\title{
Orientaciones filosóficas en la intervención fonoaudiológica de voz. El desafío actual de dar el alta
}

\section{Philosophical orientations in voice therapy. The current challenge of discharge}

\author{
Gonzalo Inostroza-Moreno ${ }^{1}$ (1) () \\ ${ }^{1}$ Universidad de los Andes; Santiago de Chile; Chile.
}

\section{Correspondencia \\ Gonzalo Inostroza-Moreno. Email: gonzalo.inostroza.moreno@gmail.com}

\section{Citar así}

Inostroza-Moreno, Gonzalo. (2021).

Orientaciones filosóficas en la intervención fonoaudiológica de voz. El desafío actual de dar el alta. Revista de Investigación e Innovación en Ciencias de la Salud. 3(1): 116-124. https://doi. org/10.46634/riics.34

\section{Recibido: 01/09/2020}

Revisado: $13 / 10 / 2020$

Aceptado: $21 / 10 / 2020$

\section{Editora invitada}

Lady Catherine Cantor-Cutiva, Ph.D.

\section{Editor in chief}

Jorge Mauricio Cuartas Arias, Ph.D. (iD

\section{Coeditor}

Fraidy-Alonso Alzate-Pamplona, MSc. iD

Copyright $($ C 2021. Fundación Universitaria María Cano. La Revista de Investigación e

Innovación en Ciencias de la Salud proporciona acceso abierto a todo su contenido bajo los términos de la licencia Creative Commons Attribution-NonCommercial-NoDerivatives 4.0 International (CC BY-NC-ND 4.0).

Declaración de intereses

El autor ha declarado que no hay conflicto de intereses.

\section{Disponibilidad de datos}

Todos los datos relevantes se encuentran en el artículo. Para mayor información, comunicarse con el autor de correspondencia.

\section{Resumen}

El presente artículo corresponde a una reflexión sobre las orientaciones filosóficas en la terapia vocal actual. Cuando existe alguna alteración o trastorno vocal, se habla comunmente de un desequilibrio entre los subsistemas involucrados en el proceso fonatorio, es decir, fuelle (sistema respiratorio), fuente (pliegues vocales) y filtro (tracto vocal). Si no hay un correcto balance, entonces el sistema no tiene un correcto funcionamiento y pueden aparecer síntomas como ronquera, sensación de cuerpo extraño, prurito, fatiga vocal o bien disfonía o incluso afonía. Como consecuencia de estas dificultades, se generan compensaciones que durante el proceso de la intervención fonoaudiológica el profesional debe volver a "equilibrar". Para lograr este objetivo, se desarrollan y analizan las diversas herramientas que entregan las filosofías de pensamiento a lo largo de la historia de la rehabilitación vocal, donde se encuentran la orientación higiénica, psicológica, sintomatológica, fisiológica y ecléctica. En este mismo sentido, el profesional debe buscar lo que percibe como más idóneo para cada paciente o grupo a intervenir, centrado en mejorar la calidad vocal y las necesidades actuales de estos, basándose en el contexto mundial actual, con el objetivo de lograr un buen proceso de entrenamiento o rehabilitación y finalmente lograr el alta.

\section{Palabras clave}

Intervención; terapia vocal; rehabilitación; orientaciones filosóficas; dar el alta; vocología; fisiología; sintomatología; higiene vocal; ecléctico; fonación; voz.

\section{Abstract}

The present article corresponds to a reflection about the philosophical orientations in the current vocal therapy. When there are some vocal alterations or disorders, we usually talk about an imbalance between the sub-sysmtems involved in the fonatory process, which are bellows (respiratory system), source (vocal folds) and filter (vocal tract). When there is not a correct balance, the system does not work properly and, 


\section{Financiamiento}

Ninguno. Esta investigación no recibió ninguna subvención específica de agencias de financiamiento en los sectores público, comercial o sin fines de lucro.

\section{Descargo de responsabilidad}

El contenido de este artículo es responsabilidad exclusiva del autor y no representa una opinión oficial de su institución ni de la Revista de Investigación e Innovación en Ciencias de la Salud.

\section{Agradecimientos}

A mis pacientes, familia y demás personas que apoyan mi trabajo y hacen posible lo que soy. as a consequence, it is possible to experiment symptoms such as hoarseness, foreign body sensation, pruritus, vocal fatigue, dysphonia or even aphonia. As a result of these difficulties, it is possible to generate compensations through the intervention of speech therapy. By virtue of this therapy the professional must 'balance'. In order to achieve this, it is necessary to develop and analyze the different tools that philosophical studies offer through the history of vocal rehabilitation. Some of these tools are hygienic orientation, psychological, symptomatological, physiological and eclectic. Thinking on that, the professional must search what it is most suitable for every patient or group of people that he/she will be working with. This must be focus on improving vocal quality and the actual necessities of the patient, based on the current global context. The final purpose of the professional is to achieve a good and healthy process of training and/or rehabilitation to accomplish medical discharge.

\section{Key words}

Intervention; voice therapy; rehabilitation; philosophical orientations; discharge; vocology; physiology; symptomatology; vocal hygiene; eclectic; phonation; voice.

\section{Introducción}

En todas las áreas de la Fonoaudiología existen diversas formas, técnicas, modelos o pensamientos filosóficos a la hora de generar un plan de tratamiento para un individuo. Las intervenciones son programas que están diseñados para solucionar los trastornos o las enfermedades específicas de cada paciente. El éxito de una intervención en particular se basa en su proceso para lograr el alivio o recuperación de los signos o síntomas asociados a una enfermedad o trastorno. La extensa evaluación en el diagnóstico de la voz le permite al fonoaudiólogo obtener un claro panorama y también las respuestas a las causas de los problemas de voz de un individuo, además de una descripción de los síntomas vocales, pudiendo posteriormente generar un plan de acción pertinente para poder satisfacer las necesidades vocales actuales. En el presente artículo se desarrollan las orientaciones filosóficas en la terapia vocal, exponiendo las diversas formas de abordar un paciente. Por ejemplo, para algunas patologías el trabajo puede constar de cinco a seis sesiones de rehabilitación durante dos meses aproximadamente y en otras hasta veinte sesiones durante seis meses [1]. Cada uno de esos resultados podrán tener variaciones según la forma en que se aborde al paciente, con base al conocimiento del terapeuta y su experiencia, además de otros factores del propio paciente.

\section{Reflexión}

En la terapia fonoaudiológica de voz hay técnicas, programas o filosofias de pensamiento que actualmente evidencian cambios a corto plazo en la voz de un individuo y otras que los generan a largo plazo. Todos estos enfoques u orientaciones se han visto afectados en la actualidad de alguna u otra manera por la contingencia mundial de COVID-19. En este contexto, la terapia de la voz enfrenta grandes desafios en la intervención a través de la telepráctica [2]. Desde hace muchos años, la forma de intervención vocal ha sido bastante interesante en cuanto a su evolución, dando lugar en sus inicios a distintos tipos de orientaciones filosóficas en la terapia. Thomas y Stemple [3] clasifican en el año 2007 estas orientaciones en cuatro grandes categorías: higiénica, sintomatológica, psicológica y fisiológica. Comúnmente, cuando se busca rehabilitar a un individuo, se quiere lograr un equilibrio entre los tres subsistemas in- 
volucrados en el proceso fonatorio: fuelle, fuente y filtro. Esta compleja y sutil sinergia entre los subsistemas de producción de la voz, y del cuerpo considerado como un macrosistema, están estrechamente relacionados con los aspectos psicoemocionales que suelen estar en el trasfondo de una alteración vocal funcional o ante la presencia de lesiones benignas adquiridas [4].

Como fonoaudiólogos, es muy relevante ver la dinámica o funcionamiento vocal desde la fisiología, basados en la evidencia científica y fortalecidos con la experiencia terapéutica, además del entendimiento emocional y psicológico del usuario [5]. Cuando hablamos de rehabilitación fonoaudiológica de voz, tenemos que pensar en dos aspectos fundamentales para generar un cambio directo en la fisiología, a saber, el cuidado de la voz (terapia vocal indirecta) y el uso de la voz (terapia vocal directa) [5]. Una de las actuales revisiones de las líneas filosóficas de intervención la clasificó Joseph Stemple (parafraseado por Guzmán) el año 2000 y las presenta como:

enfoque higiénico (relacionado con el abuso y mal uso vocal y la eliminación de estos factores), sintomatológico (relacionado con la modificación directa de síntomas), fisiológico (relacionado con la modificación de la actividad fisiológica inadecuada), psicológico (identificación y modificación de disturbios emocionales y psicosociales) y ecléctico (cuando existe una combinación de 2 o más enfoques). [6 para2]

Como su nombre lo dice, la tendencia higiénica se centra en la mejora de la salud de la cubierta de las cuerdas vocales y los tejidos laríngeos circundantes [7]. La terapia vocal higiénica es a menudo el primer paso en muchos de los programas terapéuticos. Algunos ejemplos de comportamientos representativos de una mala higiene vocal incluyen un exceso de voz a alta intensidad, tos, carraspeo excesivo y mala hidratación. Cuando se identifican los comportamientos inapropiados, se proporcionan tratamientos y recomendaciones que modificarán o eliminarán la afección y darán como resultado una mejor salud y medio ambiente de la laringe [7]. Una vez modificada, la producción vocal brinda la oportunidad de mejorar o retornar a la normalidad. En relación a los pacientes que consultan en fonoaudiología, específicamente para los artistas, probablemente lo más difícil de implementar sea la terapia vocal indirecta o higiene vocal, ya que abarca aspectos que no se consideran tan relevantes o directamente implicados con la voz, sobre todo en artistas sin estudios formales. Los resultados sugieren que los programas aislados de higiene vocal no generan cambios en la calidad vocal ni en los hábitos [8].

Otra tendencia que en la actualidad ofrece una gran gama de evidencia y de artículos publicados en diversas revistas de renombre corresponde a la tendencia fisiológica. En este marco, sugieren que este enfoque involucra tres componentes importantes:

1) Mejorar el equilibrio entre los principales subsistemas involucrados en la producción de voz: respiración, fonación y resonancia (configuración del tracto vocal y sensaciones relacionadas con la "colocación vocal"); 2) mejorar la fuerza, el equilibrio, el tono y la resistencia de los músculos laríngeos; y 3) desarrollar una cubierta mucosa de los pliegues vocales sana. La evidencia sugiere que los métodos fisiológicos de la terapia de voz tienen mayor sustento científico (mayor número de estudios y más alto nivel de evidencia) que otros enfoques de la terapia de voz. [9 p22]

Hay un punto de vista en el que convergen los programas de terapia de voz fisiológicos y es que se basan en ejercicios con tracto vocal semiocluido (TVSO). Este grupo de ejercicios incluye: 
fonación de consonantes fricativas sonoras, consonantes nasales, vocales cerradas, vibración labial y lingual, raspberry (vibración labial y lingual al mismo tiempo), la fonación en diferentes tubos con el extremo libre, ya sea libre en el aire o sumergido en un recipiente con agua, técnica de la mano sobre la boca, fonación dentro de una máscara semiocluida de ventilación y fonación en vaso de polietileno con orificio. [10 p 77]

Todos estos ejercicios tienen incidencia directa en favorecer la presión transglótica, logrando que esté suficientemente baja y haciendo que la fonación sea más económica en términos de preservar y proteger los pliegues vocales de poderosas colisiones, además de que proporciona la sensación de lograr un máximo resultado, alcanzado con un mínimo esfuerzo [11]. Cabe mencionar que estos ejercicios deben ser siempre guiados por un profesional, que entregará tareas fonatorias específicas para que las ejecute el paciente, con base a las necesidades comunicativo vocales propias de la persona, teniendo en cuenta el aprendizaje sensoriomotor, manera clave de enseñar estas técnicas, lo que permite un real aprendizaje a largo plazo y transferencia de la conducta vocal a diversos contextos.

Por otro lado, la orientación filosófica sintomática o sintomatológica se centra en la "modificación de los síntomas vocales alterados relacionados con el tono, la intensidad y la calidad vocal. Esta tendencia se basa en la creencia de que la modificación y corrección de las características de fonación, respiración y resonancia de manera aislada producen una mejora en la condición vocal" [9 p23]. Una vez identificado el mal uso vocal (conductas que resultan en un uso técnico ineficaz de la voz) y/o abusos (formas de vocalización ineficaces que tienen una mayor tendencia a dañar la mucosa de las cuerdas vocales a través del fono trauma), se eliminan o reducen a través de diversas técnicas que facilitan la terapia [7]. Cuando un paciente utiliza una de estas técnicas, le permite producir fácilmente una voz mejorada. En conclusión, la idea principal de esta orientación filosófica es la modificación directa de los síntomas vocales alterados, con el fin de minimizar el fonotrauma y la técnica vocal ineficiente mediante la aplicación de técnicas facilitadoras $[7,12]$. Los síntomas desviados pueden incluir un tono demasiado alto o bajo, una voz demasiado suave o fuerte, una fonación entrecortada o el uso de ataque vocal duro. Al realizar ejercicios directamente hacia un síntoma (forma aislada), se trabaja la memoria declarativa, lo cual va a favorecer el aprendizaje a corto plazo, poniendo algo de dificultad al aprendizaje de una correcta técnica vocal.

En otro orden de ideas, está bien entendido que las emociones, personalidad, y desorientaciones psicológicas pueden jugar un rol vital en el desarrollo y mantenimiento de desórdenes vocales. Además, es necesaria la investigación en esta área, por la contribución de las emociones y la personalidad en los desórdenes vocales relacionados con un tratamiento directo. Algunas patologías vocales tienen un gran componente emocional asociado, sobre todo las que son de origen psicógeno. En el mismo sentido, se menciona que los trastornos de la voz de tipo funcional tienen algún porcentaje de dificultades psicológicas asociadas. La disfonía psicógena generalmente puede reflejar problemas relacionados con la muerte de un miembro de la familia [13]. En general, se acepta que el trastorno psicógeno de la voz es el resultado del estrés psicosocial. Sin embargo, son pocos los estudios sistemáticos de los factores etiológicos en esta condición. Adicionalmente, aunque se estima que los efectos inmediatos de la terapia son buenos, las recaídas son frecuentes y se desconocen los efectos a largo plazo de la terapia [14]. Andersson y Schalen [14] informaron que los conflictos interpersonales relacionados con la familia y el trabajo son de fundamental importancia para acelerar esta condición. 
Cuando la calidad vocal se deteriora en ausencia de factores anatómicos y neurológicos, debe sospecharse un trastorno funcional de la voz. Como resultado, el término disfonía músculo tensional (DMT) se ha convertido en el término preferido para la disfonía funcional [15]. Aquí la importancia de que el/la fonoaudiólogo/a maneje herramientas desde una orientación filosófica psicogénica, con habilidades blandas desarrolladas, y así generar buena adherencia a la terapia, confianza por parte del paciente, que se sienta en un lugar cómodo que le permita expresarse, aplicando herramientas psicológicas de trabajo con personas. En ocasiones, es imprescindible la evaluación de forma paralela por un/a psicólogo/a. La terapia de voz es un método de tratamiento eficaz para un trastorno psicógeno de la voz. Sin embargo, en el seguimiento a largo plazo, se observa que la recaída de la enfermedad es mayor entre los pacientes que no siguieron la recomendación de evaluación psicológica [16]. En este mismo ámbito, hay patologías que merecen el análisis del origen de la problemática vocal y el fonoaudiólogo debe ser capaz de reconocerlo y saber intervenir sobre aquello, o bien realizar una derivación médica con el fin de regular la etiología del trastorno vocal, yendo directo a lo que originó la problemática inicial e intentando eliminar las causas que han producido la lesión. Lo anteriormente expuesto no siempre es factible, pero cuando se logra, la recidiva es casi nula [6].

Como última orientación filosófica tenemos la que lleva por nombre ecléctica, y cuando hablamos de algo ecléctico, nos referimos a un tipo de pensamiento u obra que puede reunir varias ideas o tendencias de diversos sistemas. Es en este punto que esta tendencia nos permite experimentar la rehabilitación vocal, utilizando más de una orientación filosófica. Se plantea que un programa de tipo ecléctico de rehabilitación vocal comprende la educación del paciente, junto con la implementación sistemática de actividades o técnicas que disminuyan los síntomas o bien permitan un correcto equilibrio entre los tres subsistemas ya mencionados. $\mathrm{Al}$ mezclar más de uno o dos enfoques de terapia, se podría promover un mejor pronóstico para el paciente [17]. La orientación ecléctica es la que busca la producción de una mejor voz y una comunicación más efectiva. Es la mejor opción, pero necesita de un amplio conocimiento por parte del terapeuta. Con la experiencia, los terapeutas de la voz sin duda podrán ir captando que lo más utilizado y recomendado es basarse en el eclecticismo, adaptarse a cada usuario, sus necesidades, sus contextos, sus pensamientos y sus gustos, elementos que siempre se deben considerar en un plan de tratamiento que permita decidir lo que se debe aplicar, sin dejar de lado la ciencia que sustenta el ejercicio escogido para el entrenamiento o la rehabilitación vocal de ese individuo.

No cabe duda que todos los enfoques tienen sus ventajas y desventajas, por tanto se debe ser capaz de ser crítico frente a cada uno de ellos y reflexionar sobre la razón de utilizar uno por sobre otro [6]. Así, "No hay que buscar 'recetas de cocina', muchas veces encontraremos pacientes con patologías idénticas y que sin embargo no responden de la misma forma ante un mismo recurso; cada paciente es un ser único con una situación personal y con necesidades particulares" [18 p6].

Es muy importante conocer con certeza estas orientaciones filosóficas de terapia, las cuales se pueden implementar de forma individual, como también de forma grupal, en caso de que se trabaje por ejemplo en escuelas de teatro, conservatorios de música (con cantantes), u otros lugares donde el trabajo se desarrolle de forma grupal. De hecho, algunos terapeutas prefieren realizar trabajos de rehabilitación grupales, o bien muchas veces no hay otra alternativa, como señala Coll et al. [18]: 
De ser así deben realizarse grupos lo más homogéneos posibles, con las mismas necesidades terapéuticas (no se puede mezclar, por ejemplo, un nódulo donde se deben realizar ejercicios de relajación, con una parálisis donde debe hacerse exactamente lo contrario: ejercicios de fuerza), tampoco deben mezclarse hombres con mujeres porque la zona vocal a ejercitar es diferente; ni niños con adultos porque la forma de trabajo es distinta. No se aconsejan más de cuatro personas por grupo. [18 p5]

El hallazgo de Ohlsson et al. [19] muestra una mejora a largo plazo de la terapia conductual de la voz, particularmente en un entorno grupal. Los resultados indican la importancia de la transferencia temprana al habla y la prueba de posterapia para confirmar si el objetivo de la terapia de voz se cumplió o no para los pacientes [19]. Sin duda, para fortalecer la etapa de la transferencia o de generalización de la conducta, como también es conocida, se favorece la terapia grupal, aunque se aleja del trabajo minucioso de las necesidades vocales particulares de cada persona.

En la actualidad hay mayor conciencia de los problemas vocales, existen más cantidad de profesionales donde acudir, hay mayor conocimiento respecto a los trastornos de voz y de su rehabilitación, y también hay mayor cantidad de personas que sufren dificultades en su voz. Los problemas de voz en Estados Unidos son un problema de salud pública importante; su prevalencia es tan alta como un 30\% de la población general, pero mucho más alta en usuarios profesionales de la voz, es decir, que utilizan su voz como herramienta de trabajo [20, 21]. La disfonía severa, por ejemplo, es una condición muy fatigante con un impacto negativo en la vida social y profesional. Al presente, la era de la pandemia de la enfermedad del coronavirus 2019 (COVID-19), el bloqueo y las restricciones sociales, han provocado una interrupción abrupta de los programas de terapia de la voz. Esta falta de atención puede incidir en la posibilidad de restablecer una voz socialmente aceptable en un tiempo razonable, teniendo un posible impacto negativo en la carrera profesional y la vida emocional [22]. A pesar de la adversidad actual, se han podido concluir terapias y darle el alta al paciente, utilizando todas las herramientas que pueden brindar las orientaciones filosóficas y permitiendo que estos adquieran un uso de la voz favorable. Los fonoaudiólogos están de acuerdo en que la capacidad de un paciente para utilizar técnicas de voz en conversaciones y situaciones de la vida real fuera de la sala de terapia son los determinantes más importantes para el alta de la terapia de voz [23]. Por ejemplo, un grupo de pacientes que en general consulta por problemas de su voz, son los cantantes, por lo que identificar sus características específicas, en aquellos que completan o abandonan la terapia de la voz, puede permitir a los profesionales atender mejor sus necesidades especializadas, en el uso de sus voces de manera profesional y recreativa [24]. Es por eso que Inostroza y Zúñiga mencionan que se hace imprescindible tener un conocimiento integral sobre todos los elementos que son de utilidad al trabajar con otra persona, es decir, un individuo con características propias, experiencias, estudios, gustos, etc [25].

Para crear un plan de tratamiento acorde a estas ideas sobre el otro, debemos tener un pensamiento ecléctico, que se caracterice por la habilidad de reunir varias ideas o tendencias de diversos sistemas [25]. En este mismo sentido, Desjardins et al. [26] señalan que la terapia de voz de tipo conductual generalmente conduce a mejoras significativas en los resultados de la voz, pero se necesitan más investigaciones que consideren el significado clínico de los resultados, para establecer lo que realmente significa el término "efectividad" cuando se trata de terapia de voz [26]. Por consiguiente, un elemento de importancia previo a pensar, en la orientación filosófica que se utilizará con el paciente, es realizar un buen proceso de evaluación, incluyendo todos los aspectos necesarios: clínicos, acústicos, habilidades musicales u 
otros. La evaluación clínica del fonoaudiólogo es multidimensional y multiparamétrica, con análisis subjetivos y objetivos. La opinión de los pacientes sobre el impacto de un problema de voz en su calidad de vida es significativa a la hora de proponer un tratamiento. Por tanto, también se incluye en la evaluación inicial [27]. La evidencia del efecto positivo de la terapia de la voz ahora está bien establecida, no sólo si se habla de disfonías de tipo funcionales u orgánicas, siendo estas últimas no necesariamente el resultado de conductas vocales dañinas. También pueden beneficiarse de la rehabilitación vocal actual [28], siempre y cuando el terapeuta conozca y sea capaz de entregar las instrucciones adecuadas, ya que las expectativas del resultado de la terapia son naturalmente diferentes para los pacientes con diversos tipos de trastornos de la voz [29].

\section{Conclusiones}

Con base a todo lo anteriormente expuesto hasta aquí, se debe destacar sobremanera la necesidad de comprender por parte de los terapeutas que cada paciente es único, aunque las patologías sean las mismas o la técnica vocal que quiera alcanzar sea igual a la de otro usuario. En cada caso debemos valorar desde el proceso de evaluación hasta saber cuáles son los recursos terapéuticos más idóneos para ese sujeto en particular. En ocasiones, se podrá abordar con técnicas higiénicas, en otras necesitaremos de técnicas facilitadoras, quizás llegar a la causa de su problema, o bien trabajar todo en conjunto de manera fisiológica. En todos los casos anteriormente mencionados es importante proporcionar al paciente información sobre el problema que le aqueja, los comportamientos abusivos que impiden el trabajo correcto de su laringe, detallando para qué sirven los ejercicios que se están realizando con él, es decir, integrarlo a la terapia, que se sienta parte de ella completamente, lo que generará mayor compromiso y adherencia, traduciéndose en resultados satisfactorios. Como compromiso, queda reformular y actualizar estas tendencias, con el fin de homologar la interpretación en la terapia Fonoaudiológica, por ende, la forma de trabajo, entregando una mirada desde la inclusión y la diversidad.

\section{Referencias}

1. Formánek M, Walderová R, Baníková S, Chmelová I, Formánková D, Zeleník K, Komínek P. Effect of voice therapy with or without transcutaneous electrical stimulation on recovery of injured macroscopically intact recurrent laryngeal nerve after thyroid surgery. Eur Arch Otorhinolaryngol. 2020;277:933-938. doi: https://doi.org/10.1007/s00405020-05806-1

2. Castillo-Allendes A, Contreras-Rouston F, Cantor L, Vaiano T, Wilder F, Behlau M, et al. Voice therapy in the context of the COVID-19 pandemic: guidelines for clinical practice. Journal of Voice. 2020;1997(20):30287-3. doi: https://doi.org/10.1016/j.jvoice.2020.08.001

3. Thomas LB, Stemple JC. Voice Therapy: Does Science Support the Art? Communicative Disorders Review. 2007;1(1):49-77. Disponible en: https://tinyurl.com/yc2af3es

4. Bustos Sánchez I. Abordaje holístico y potenciación del filtro vocal en alteraciones de la voz. Areté. 2018;(2):55-64. doi: https://doi.org/10.33881/1657-2513.art.18205

5. Muckala J. Voice Rehabilitation and Resilience Work With the Commercial Singer: A Concept Piece. American Speech-Language-Hearing Association. 2020 Feb;5(1):102112. doi: https://doi.org/10.1044/2019_PERSP-19-00074 
6. Guzmán D. Enfoques Terapéuticos de la Voz: Historia y Reflexión. Pontificia Universidad Católica de Chile, 2020 [Internet]. Disponible en: https://tinyurl.com/yasctwar

7. Fastest Otolaryngology \& Ophthalmology Insight Engine. 2020 [Internet]. Disponible en: https://tinyurl.com/ycppev9x

8. Goulart BNG, Rocha JG, Chiari BM. Intervenção fonoaudiológica em grupo a cantores populares: estudo prospectivo controlado. J Soc Bras Fonoaudiol. 2012;24(1):7-18. doi: https://doi.org/10.1590/S217964912012000100004

9. Guzman M, Salfate, L. Ejercicios con tracto vocal semi-ocluido: Efectos en la función glótica, aerodinámica y configuración del tracto vocal. Areté. 2018;18(2):21-32. doi: https://doi.org/10.33881/1657-2513.art.18203

10. Manzano Aquiahuatl C. Rehabilitación fisiológica de la voz: Terapia de resistencia en el agua artículo de revisión. Areté. 2018 (2): 75-82. doi: https://doi.org/10.33881/16572513.art. 18207

11. Hampala Vít, Laukkanen A-M, Guzman M, Horáček J, Švec J. Vocal fold adjustment caused by phonation into a tube: A double-case study using computed tomography. Journal of Voice. 2015;29(6):733-742. doi: http://dx.doi.org/10.1016/j.jvoice.2014.10.022

12. American Speech-Language-Hearing Association. Voice Disorders. [Internet]. [citado el 28 de agosto 2020]; Disponible en: https://www.asha.org/practice-portal/clinical-topics/voice-disorders/

13. Garcia-Martins R, Abrantes do Amaral H, Mendes-Tavares E, Garcia-Martins M, Gonçalves T, Hernandes-Dias N. Voice Disorders: Etiology and Diagnosis. Journal of Voice. 2016;30(6):761.e1-761.e9, doi: http://dx.doi.org/10.1016/j.jvoice.2015.09.017

14. Andersson K, Schalén L. Etiology and treatment of psychogenic voice disorder: Results of a follow-up study of thirty patients. Journal of Voice, 1998;18(1):96-106, doi: https:// doi.org/10.1016/S0892-1997(98)80081-7

15. Chheda Neil N. Functional Voice Disorders. Medscape [Internet]. 16 de Julio 2018 [citado 29 de Agosto 2020]. Disponible en: https://emedicine.medscape.com/article/865191-overview

16. Çiler Tezcaner Z, Fatih Gökmen M, Yildirim S, Dursun G. Clinical features of psychogenic voice disorder and the efficiency of voice therapy and psychological evaluation. Journal of Voice. 2017;33(2):250-254. doi: https://doi.org/10.1016/j.jvoice.2017.09.022

17. P Gopikishore, M Pushpavathi. Outcomes of Eclectic Voice Therapy Program in the Treatment of Hyperfunctional Voice Disorders: A Preliminary Study. Glob J Oto. 2017;8(2):555732. doi: https://doi.org/10.19080/GJO.2017.08.555732

18. Coll R, Mora-Rivas E, Cobeta- Marco I. Fundamentos de la rehabilitación Fonatoria. Libro virtual de formación ORL, SEORL PFG, IV. Laringe Árbol Traqueo-Bronquial, Cap 119, Hospital Ramón y Cajal, Madrid 2014. [Internet], [citado 29 de Agosto 2020]. Disponible en: https://tinyurl.com/y8v2dgyd 
19. Ohlsson A, Dotevall H, Gustavsson I, Hofling K, Wahle U, Österlind C. Voice therapy outcome-a randomized clinical trial comparing individual voice therapy, therapy in group and controls without therapy. Journal of Voice. 2018;34(2):303.E17-303.E26, doi: https://doi.org/10.1016/j.jvoice.2018.08.023

20. Benninger M, Holy C, Bryson P, Milstein C. Prevalence and Occupation of Patients Presenting With Dysphonia in the United States. Journal of Voice. 2017;31(5):594-600, doi: https://doi.org/10.1016/j.jvoice.2017.01.011

21. Lyberg-Åhlander V, Rydell R, Fredlund P, Magnusson C, Wilén S. Prevalence of voice disorders in the general population, based on the Stockholm public health cohort. Journail of Voice. 2018;33(6):900-905. doi: https://doi.org/10.1016/j.jvoice.2018.07.007

22. Cantarella G, Barillari M, Lechien J, Pignataro L. The challenge of virtual voice therapy during the COVID-19 pandemic. Journal of Voice. 202018 June; Article in press. doi: https://doi.org/10.1016/j.jvoice.2020.06.015

23. Gillespie A., Gartner-Schmidt. Voice-Specialized Speech-Language Pathologist`s criteria for discharge from voice therapy. Journal of Voice. 2017;32(3):332-339. doi: https://doi. org/10.1016/j.jvoice.2017.05.022

24. Adessa M, Stadelman-Cohen T, Zipse L, Guarino A.J, Heaton J. Factors affecting voice therapy completion in singers. Journal of Voice. 2017;32(5):564-571. doi: https://doi. org/10.1016/j.jvoice.2017.06.021

25. Inostroza-Moreno G, Zúñiga-Beñaldo E. Intervención Fonoaudiológica en artistas: hacia una mirada completa de intervención vocal. Revista de Investigación e Innovación en Ciencias de la Salud. 2020;2(2):83-92. doi: https://doi.org/10.46634/riics.35

26. Desjardins M, Halstead L, Cooke M, Shaw Bonilha H. A systematic review of voice therapy: What "Effectiveness" really implies. Journal of Voice. 2016;31(3):392.E13-392.E32. doi: https://doi.org/10.1016/j.jvoice.2016.10.002

27. Casper J, Brewer D, Colton R. Variations in normal human laryngeal anatomy and physiology as viewed fiberscopically. Journal of Voice. 1987;1(2):180-185. doi: https://doi. org/10.1016/S0892-1997(87)80043-7

28. Mara B. The 2016 G. Paul Moore Lecture: Lessons in voice rehabilitation: Journal of voice and clinical practice. Journal of Voice. 2018;33(5):669-681. doi: https://doi.or$\mathrm{g} / 10.1016 / \mathrm{j}$.jvoice.2018.02.020

29. Guzmán M, Castro C, Madrid S, Olavarría C, Leiva M, Muñoz D, Jaramillo E, Laukannen A-M. Air Pressure and contact quotient measures during different semioccluded postures in subjects with different voice conditions. Journal of Voice. 2016;30(6):759.e1-759. e10. doi: https://doi.org/10.1016/j.jvoice.2015.09.010 